\title{
صورة الإسلام والمسلم الآخر في الإعلام الغربي عوامل التشكيل وسبل التغيير
}

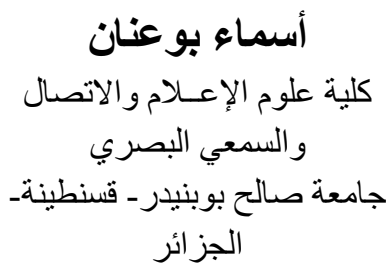

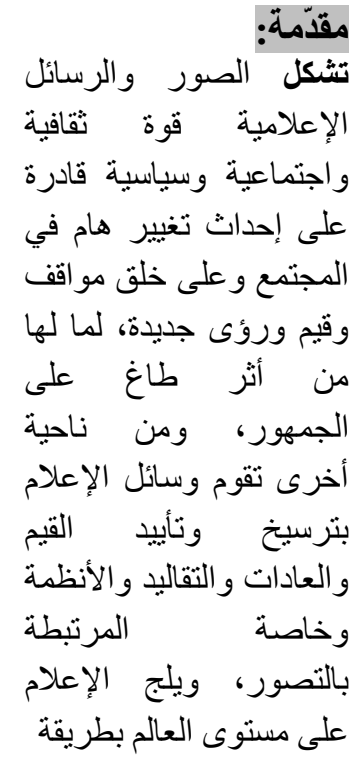

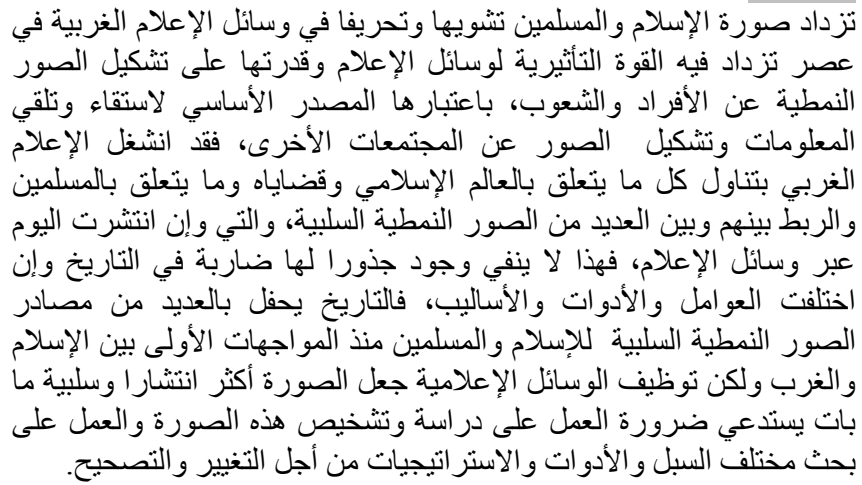

\section{Abstract:}

In an era whereby the influential power of media and their ability to shape the individuals and societies' stereotypes are increasingly omnipotent, due to their important role as an essential source of supplying and receiving information and creating images about societies, the image of Islam and Muslims is being more and more deteriorated and twisted in the western media. The latter has closely concentrated on all what concerns the Islamic world and Muslims' issues and related them to different negative stereotypes which, widely spread over the media nowadays, are collocated to different negative concepts namely terrorism. Being as such, the current fact does not refute the historical genesis of the situation. Within different contexts and using different tools and strategies, history reports abundant sources of negative stereotypes of Islam and Muslims since the early confrontations between Islam and the west. Yet, the adoption of mass media has made the image even more negative and more spread, what comes to require the necessity of studying and diagnosing this image and exploring tools and strategies to correct it.

2017 جامعة الإخوة منتوري قسنطينة، الجز ائر (C) 
واسعة إلى التميط فهو يقوم بدور الرمز الذي بقدم للجمهور المتلقي مفهوما ما، أو مفتاحا سريعا

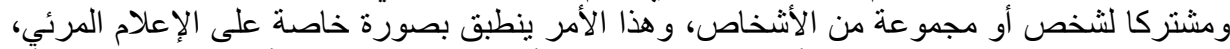

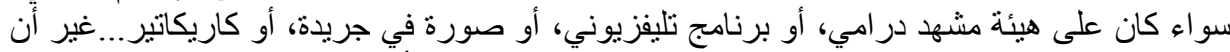

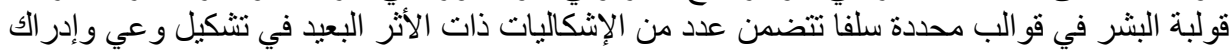

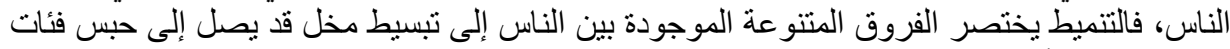

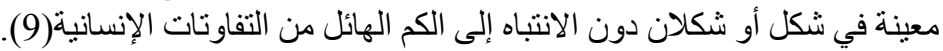

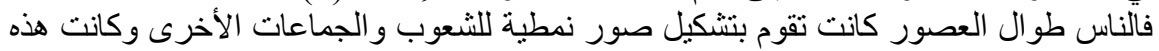

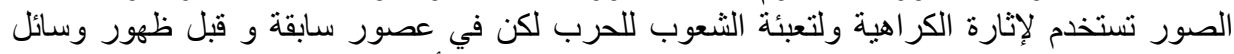

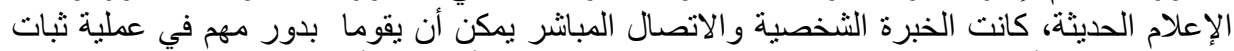

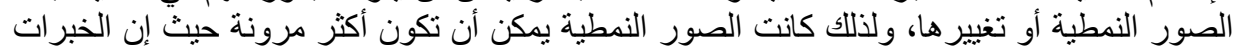

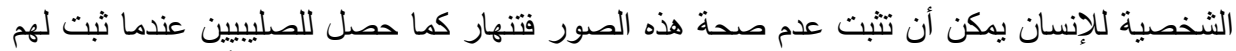

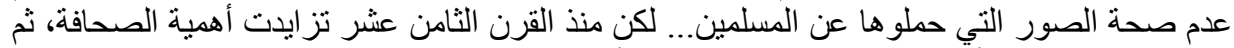

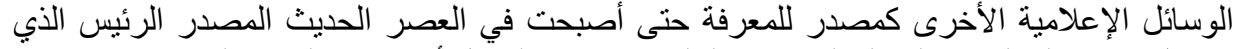

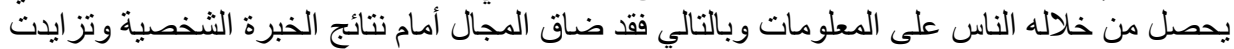
إمكانية ثبات الصور النمطية وتزايدت أهمية الدور الذي تلعبه هذه الصور في في تثكيل اتجاهات

وكما بات جليا فإن صورة العالم الإسلامي تتعرض في كل يوم لكثير من التشويه والتحريف

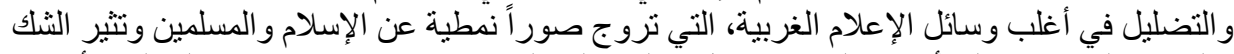

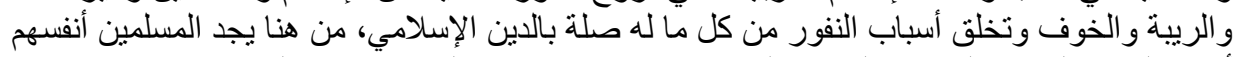

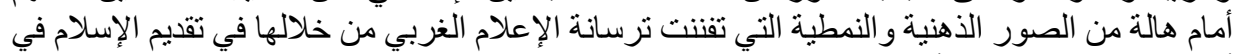

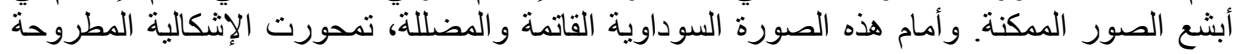

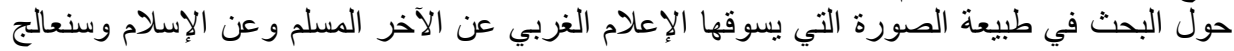

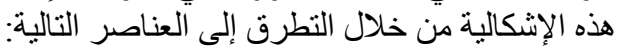
ـ ـ العودة إلى أهم العو امل والأسباب التي أسهمت في رسم ونتشويه صورة الإلئه الإسلام في الغرب.

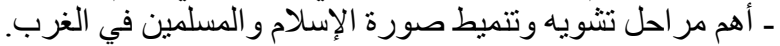

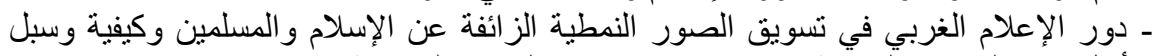
الرد من أجل تقديم الصورة الحقيقية عن الإسلام وتصحيح الصور المشو هئ عنه.

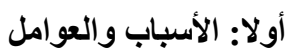

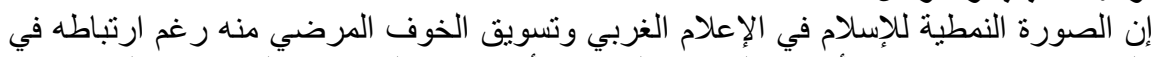

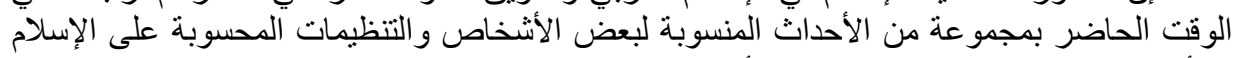

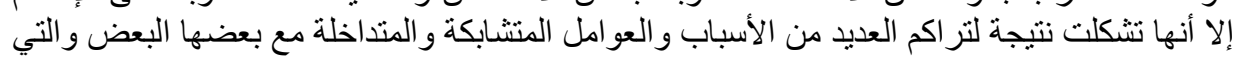

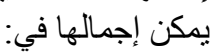
1 - العامل التاريخي والحضاري:

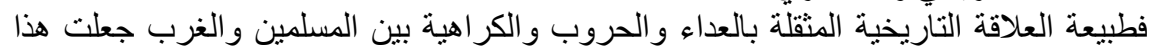

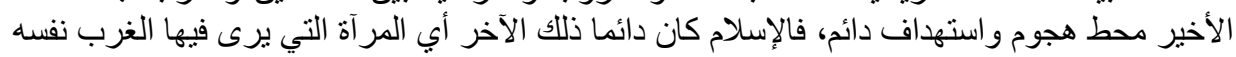

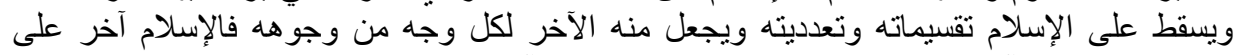

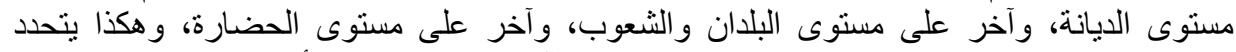

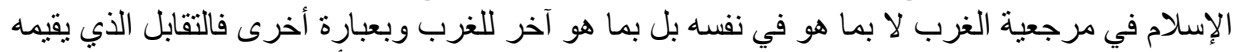

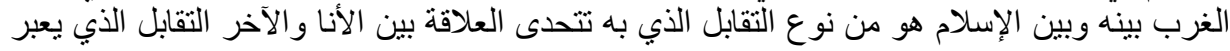


ويتبلور أساسا في المشكلة المعرفية عن الإسلام الناتجة عن جهل الغرب بكثير من حقائق الإسلام

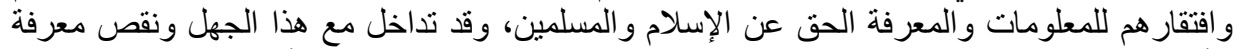

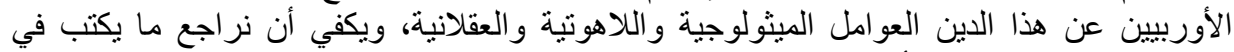

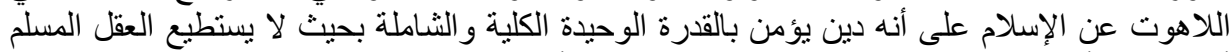

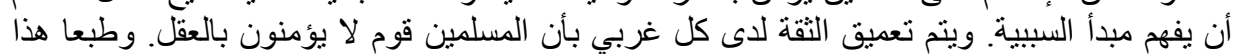

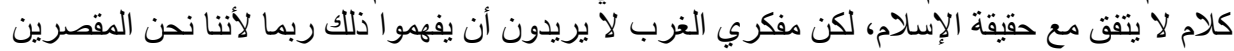

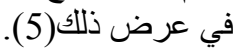

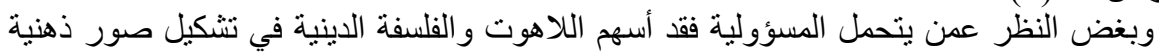

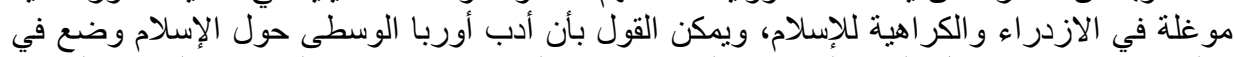

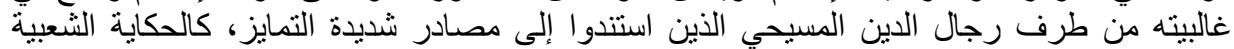

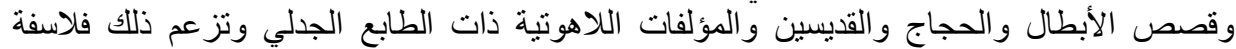

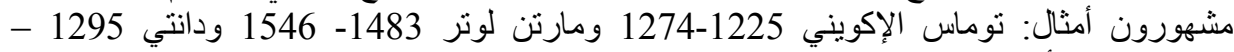

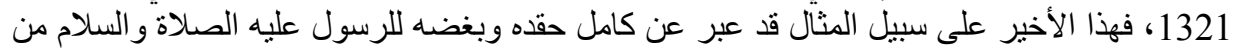

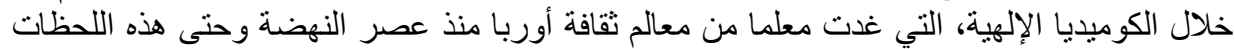

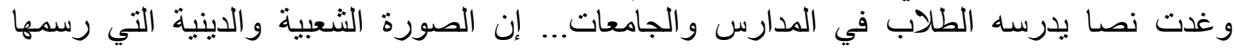

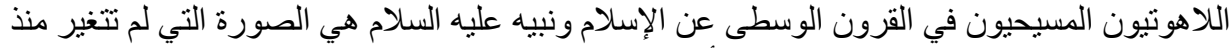

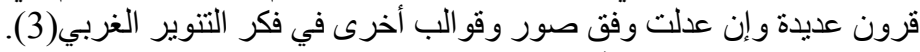

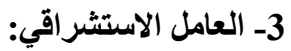

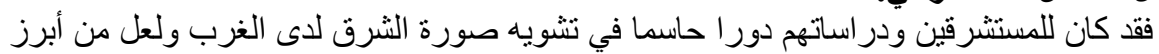

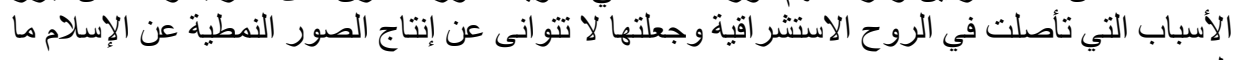

ـ ـ اعتبار الإسلام دينا منافسا للتراث اليهودي والمسيحي ولذلك يتم نفيه ورفض مش مشرو عيته.

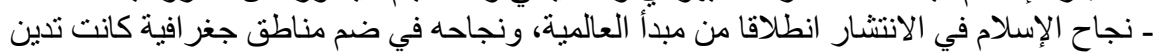

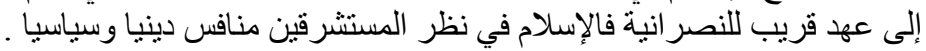

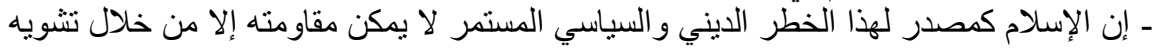
صورتهـ...قصد الحيلولة دون الإقبال على هذا الإدين.

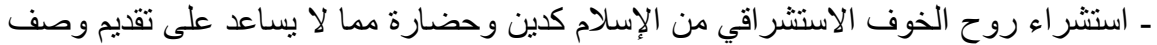

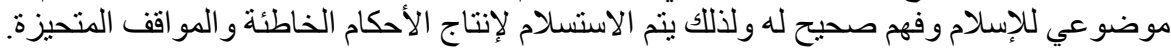

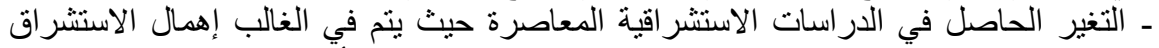

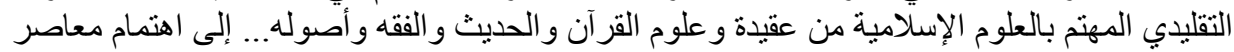

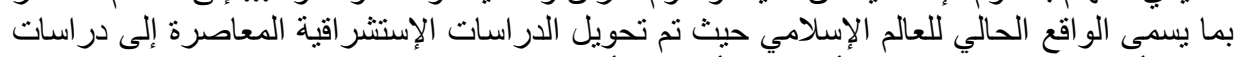

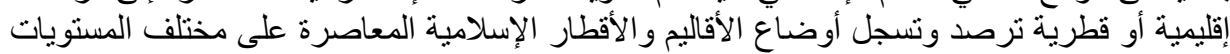

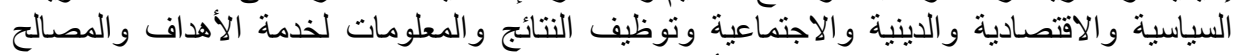

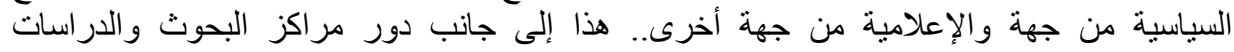

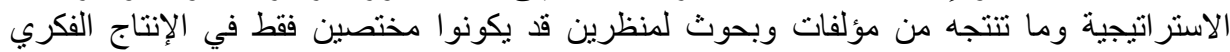

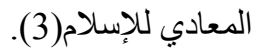

\section{-4 الحركة الاستدمارية للغرب على العالم الإسلامي:}

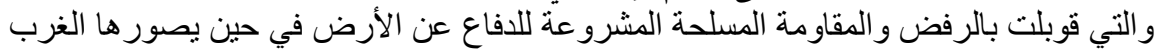

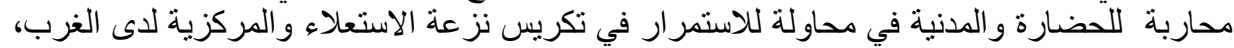

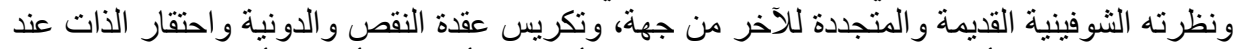
المسلمين من جهة أخرى فمعظم الغربيين يؤمنون بأنهم من أعراق وأجناس أكثر رفعة ورقئ وريا من 
المسلمين مما يزيد في تأجيج مظاهر العنصرية لدى الغرب، و التطرف لدى بعض التود الفئات من المسلمين

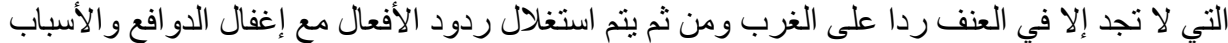

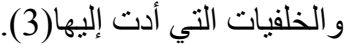

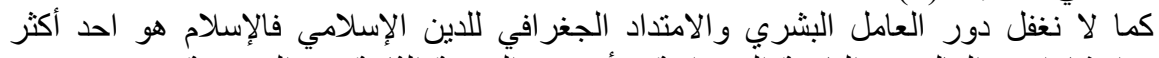

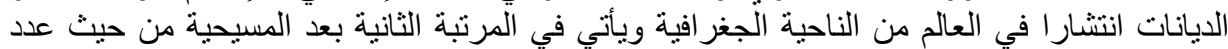

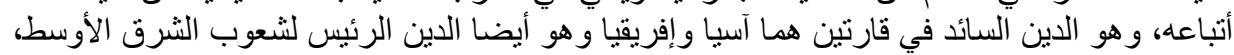

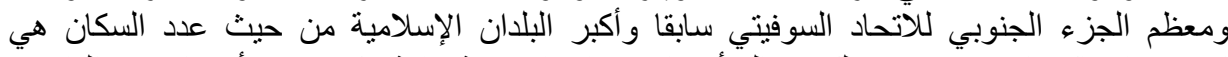

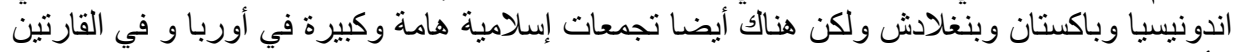

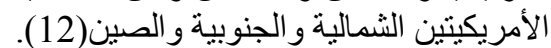

وتدل المؤشرات الإحصائية على زيادة أعداد المسلمين في مختلف دول العين العالم ولان سيما دول

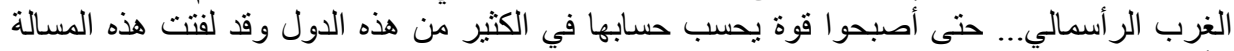

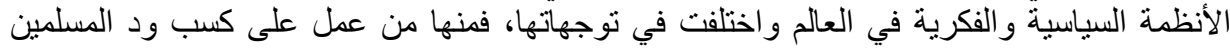

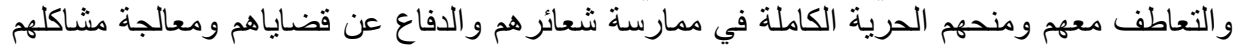

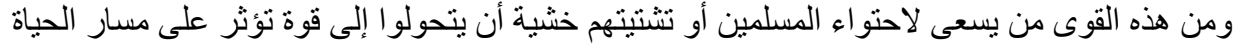
السياسية والاقتصادية في هذه الدول وراحت تثن عليهم الحملات المضادة التي تشككاتك في

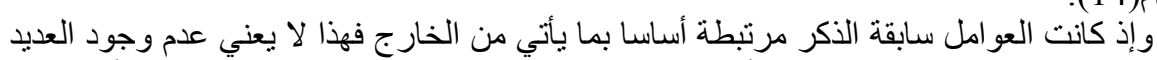

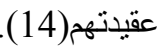

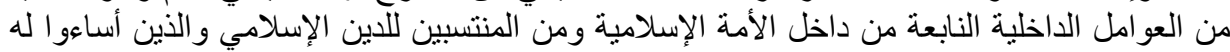

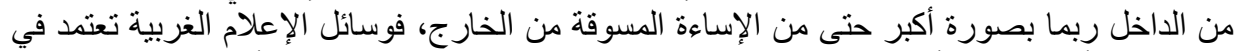

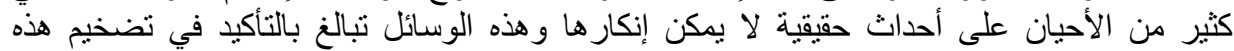

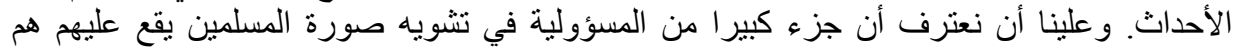

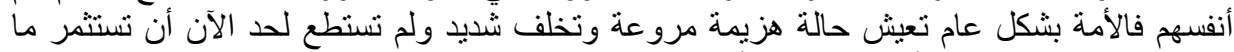

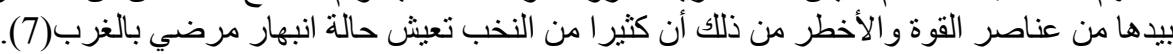

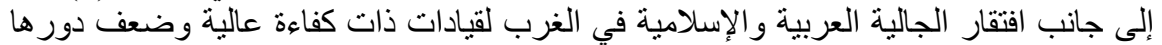

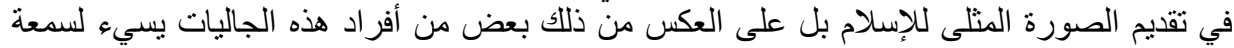

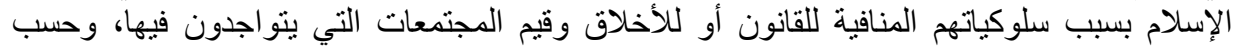

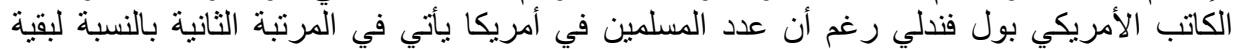

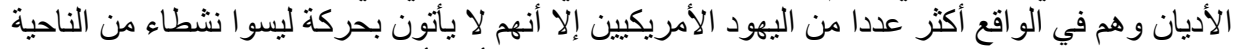

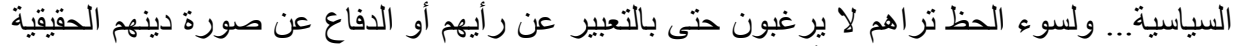

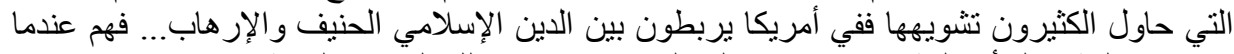

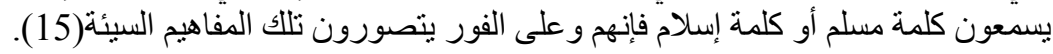

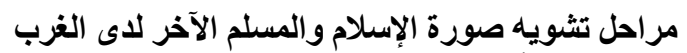

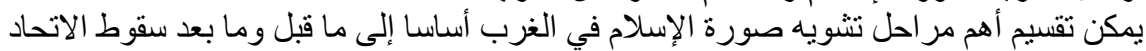

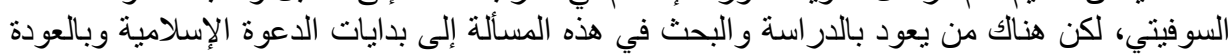

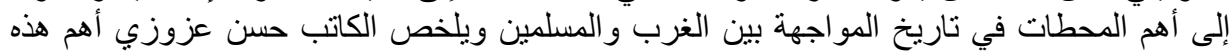

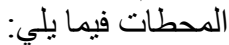

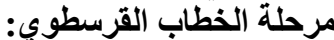

استمد فيها الغرب صورته الذهنية عن الإسلام و المسلمين من خلال المو اجهة الفعلية بين الإسلام

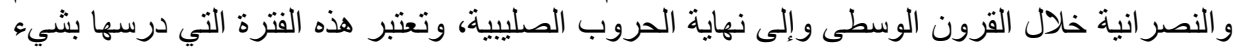

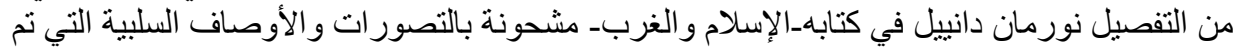
نسجها في حق الإسلام و المسلمين حيث اعتبر الدين الجديد هرطقة وسوطا من سياط العقاب الإلهي. وتم 
تصوير المسلمين وثثيين بعبدون ثالوثا مكون من: ابولين، تيرفاجونت ومحمد، والإسلام حسبهم دين إنيا

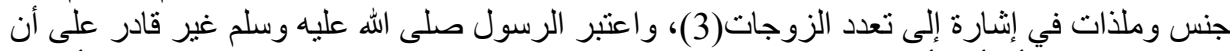

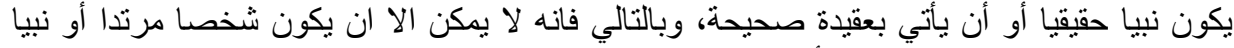

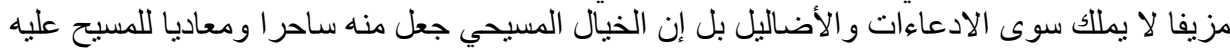

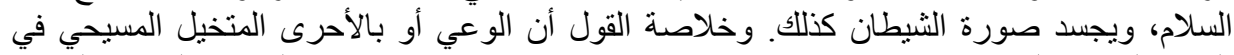

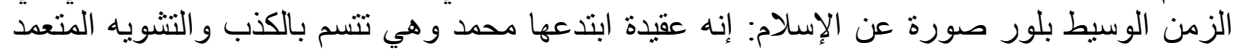
للحقائق، إنها دين الجبر و الانحلال الخلقي و التنساهل مع الملذات و الثنهوات الحسية، إنها ديانة العنف

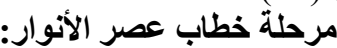

و القسوة(13).

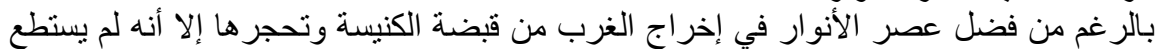

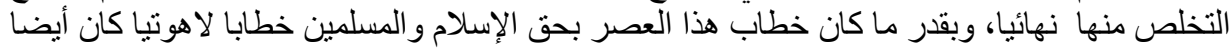

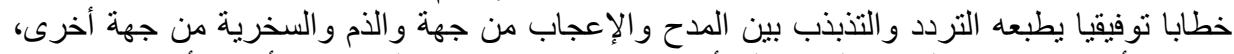

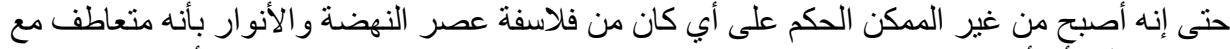

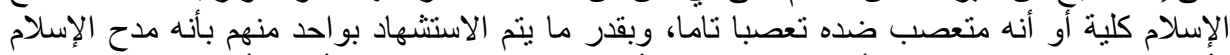

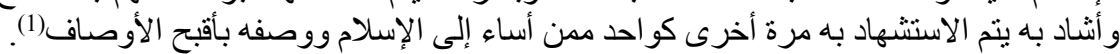

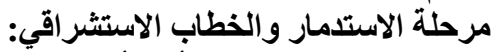

وفي هذا السباق بمكن التأكيد أيضا على أن أن واقع الاستدمار الغربي للادول الإسلامية كان منبعا

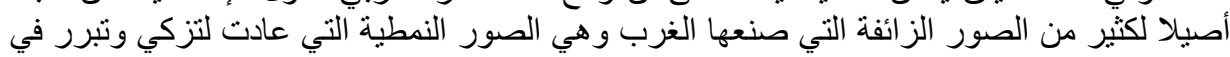

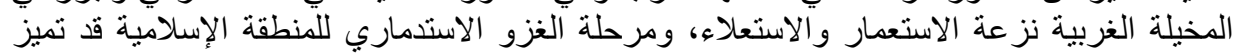

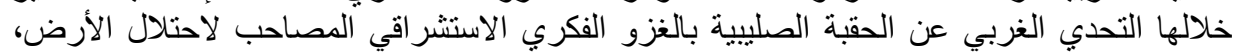

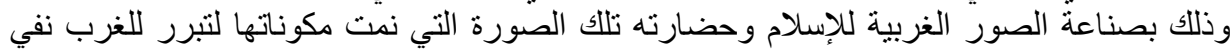

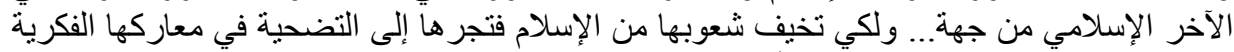
و القتالية ضد العالمي الإسلامي من جهة أخرى.

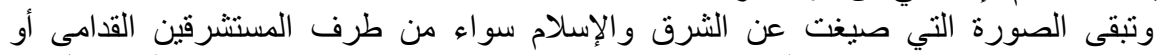

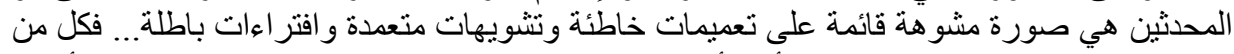

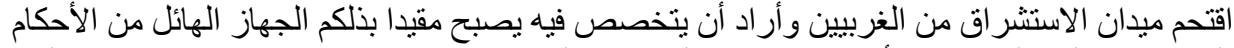

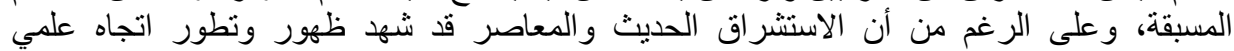

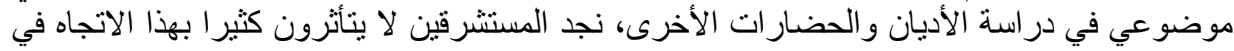

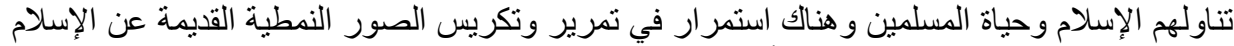

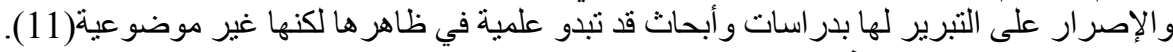
دور الإعلام الغربي في تثثكيل الصورة الصابرة

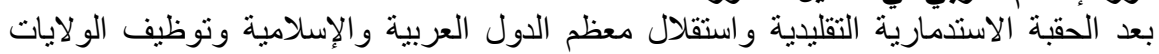

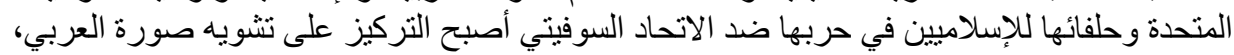

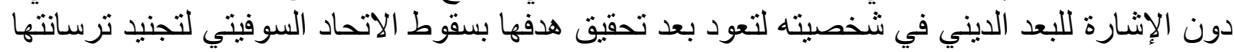

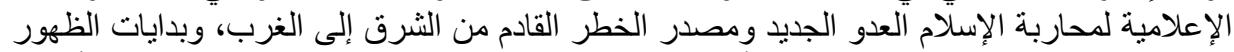

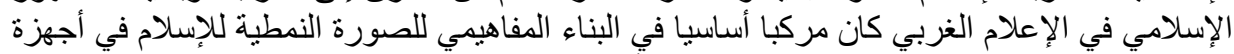

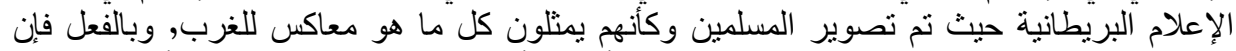

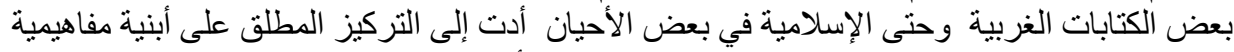

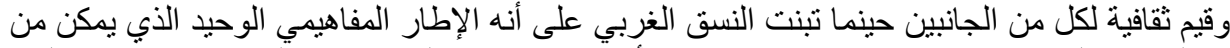

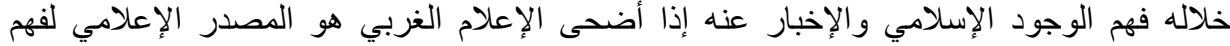

الإسلام(4). 


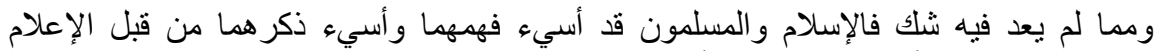

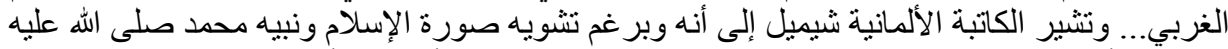

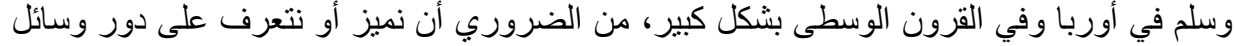

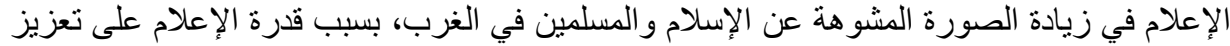
النماذج النمطية التي كانت مهمة بالفعل وذات مغزى في تعزيز الإنيز الصورة السلبية عن الإسلام و المسلمين

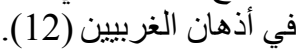

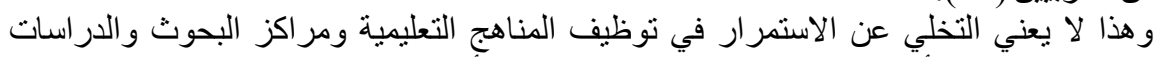

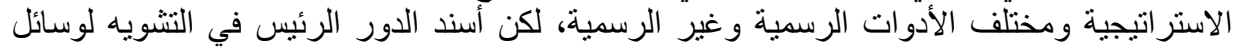

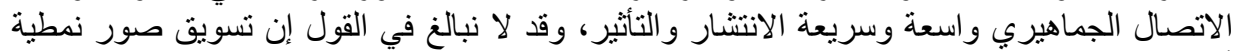

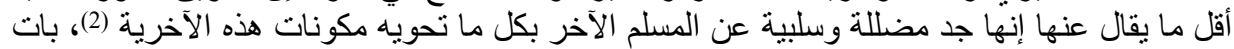
من أولى أولويات أدوات الإعلام الغربي.

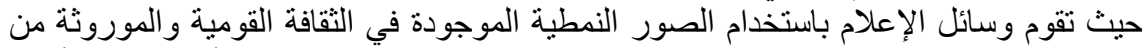

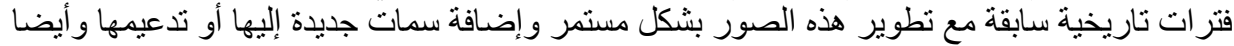

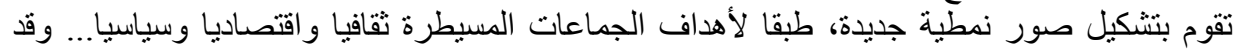

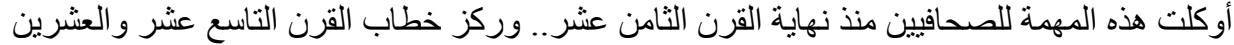

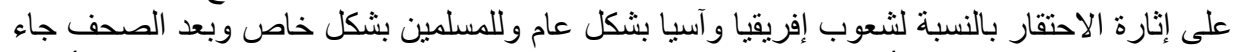

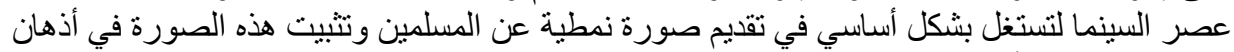

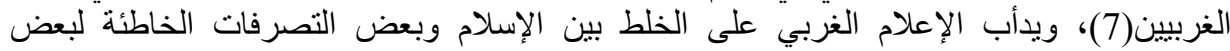

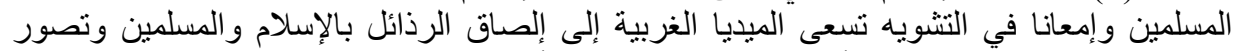

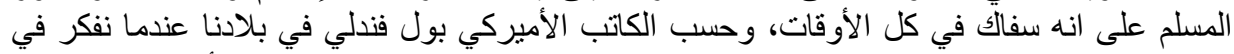

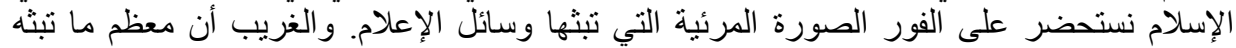

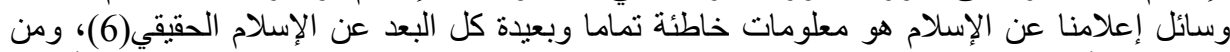

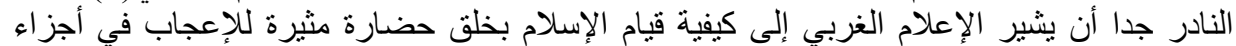

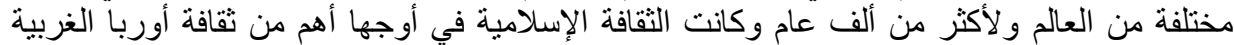

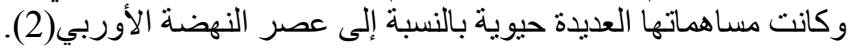

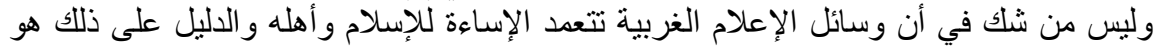

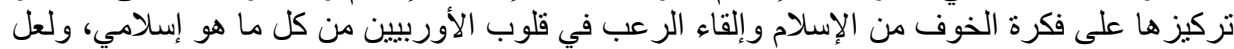

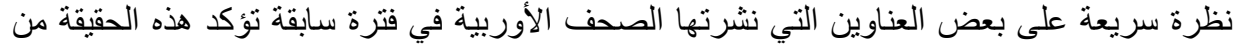

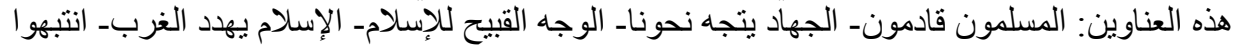

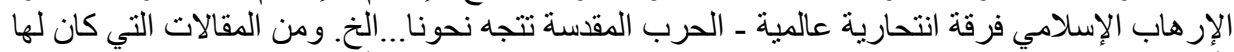

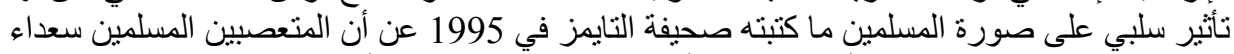

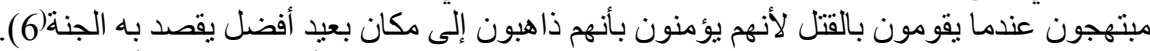

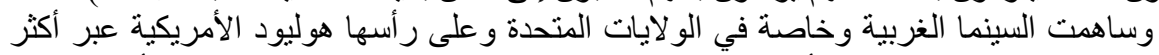

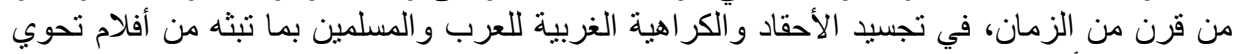

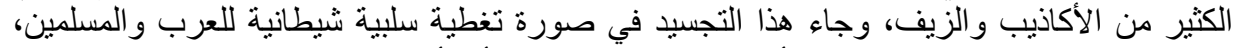

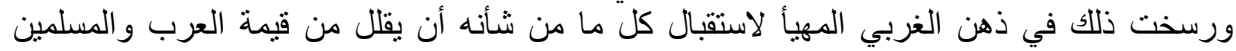

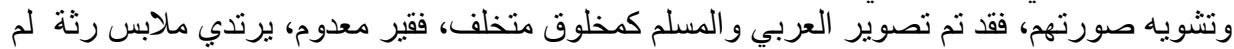

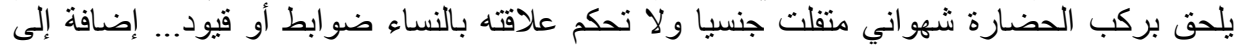

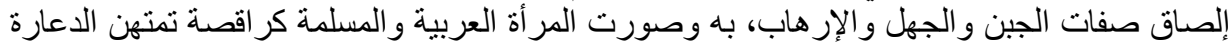

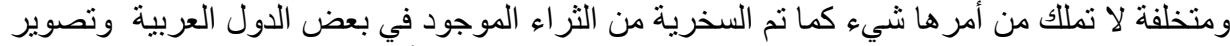

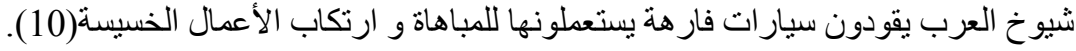


ومع أن تصوير بعض الدراسات للنهج الذي اتبعته السينما الغربية بأنه يهذف لتحقيق أرباح

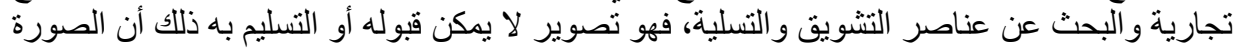

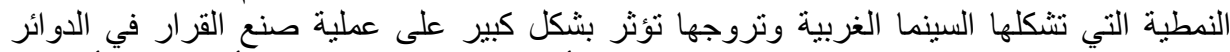

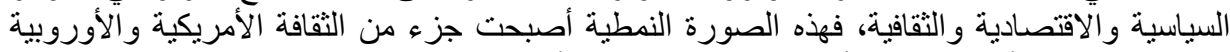

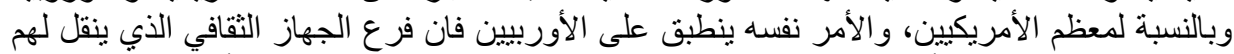

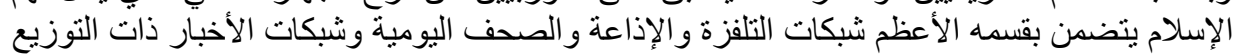

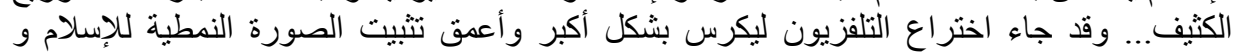

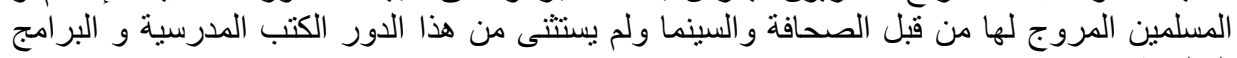

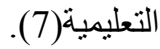

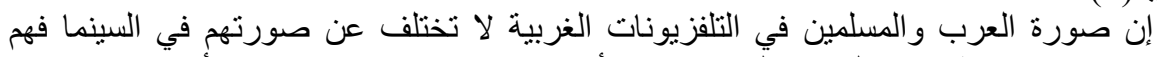

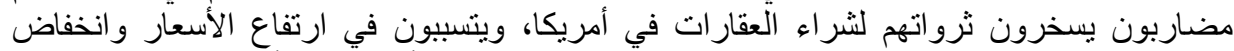

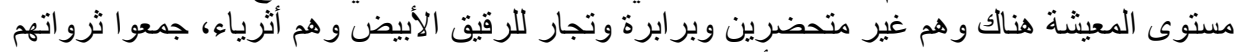

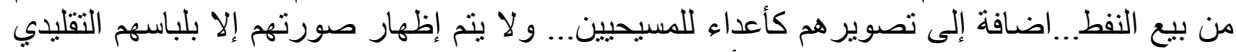

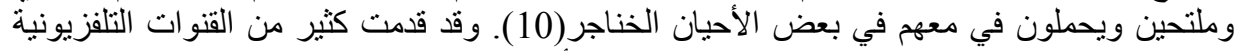

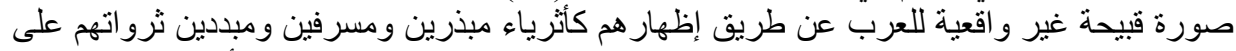

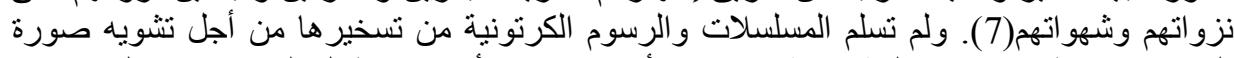

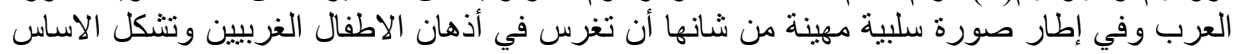
الذي يبني عليه كامل الصورة السلبية للعرب من المسلمين في المرن في الحل التالية من العمر.

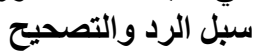

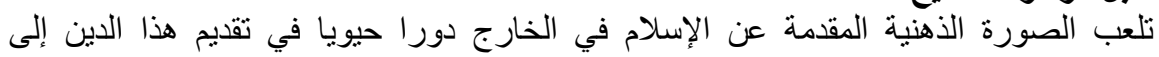

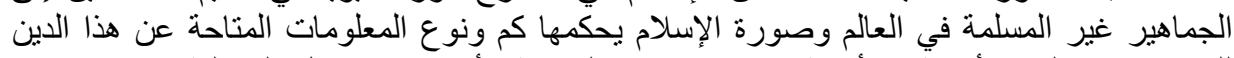

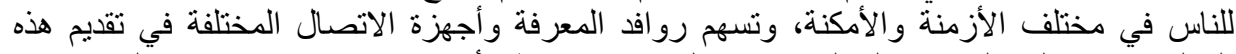

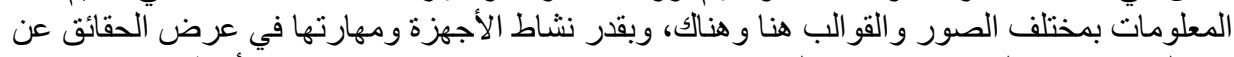

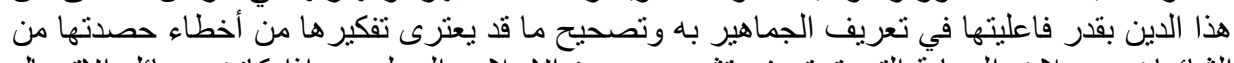

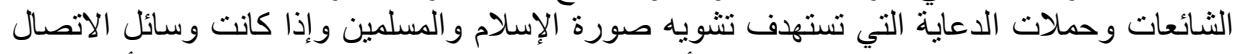

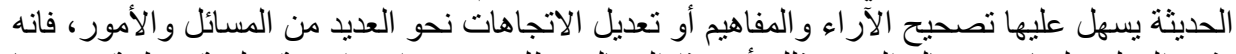

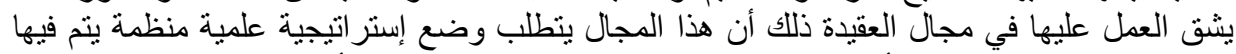

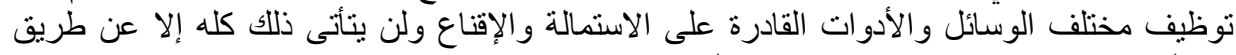

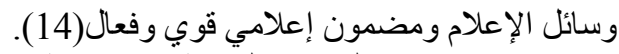

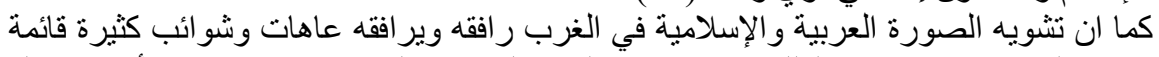

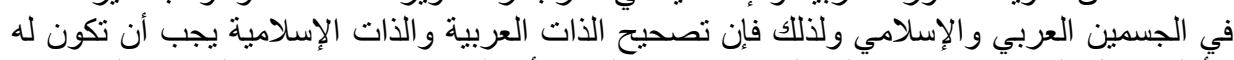

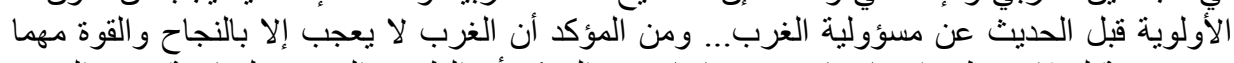

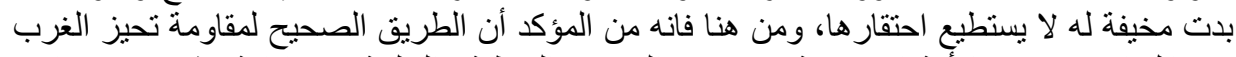

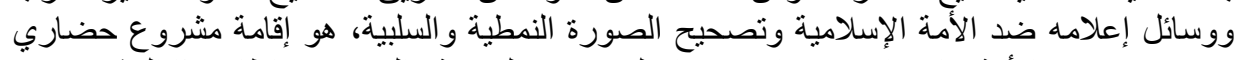

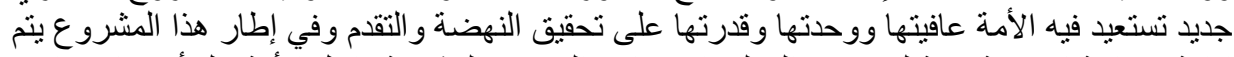

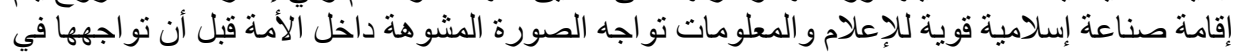

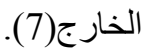

$$
\text { هذا إلى جانب ضرورة العمل على ما يلي: }
$$

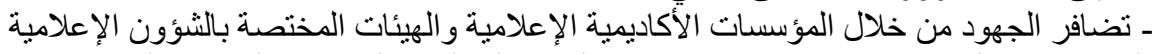

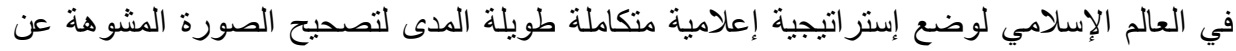
العرب و المسلمين وقضاياهم العادلة. 


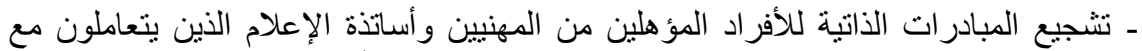

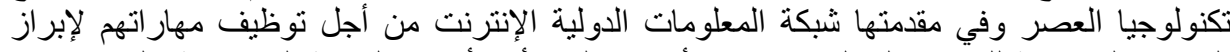

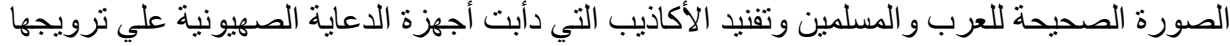

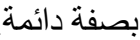

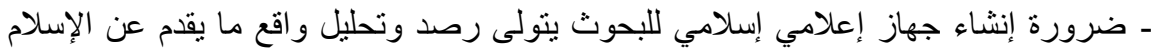

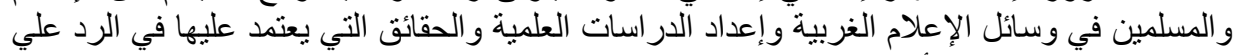

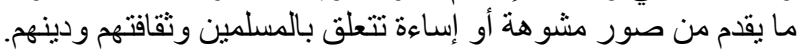

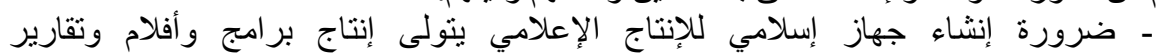

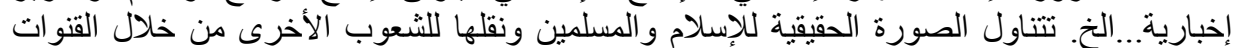

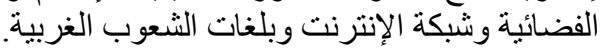

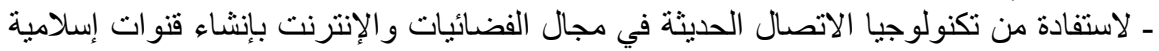

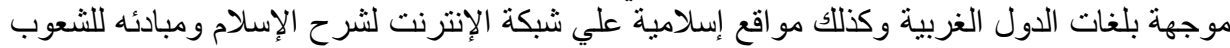

ــ إنشاء صندوق إسلامي للإنفاق علي تحسين صورة الإسلام يتم تمويله من خلال دعم الحكومات

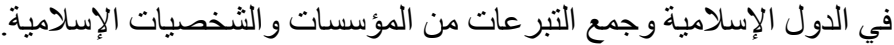

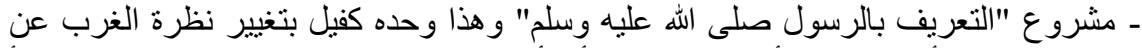

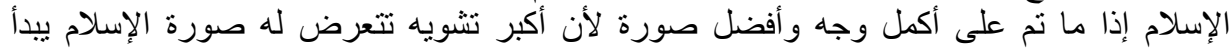

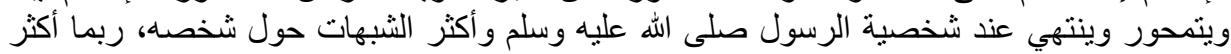

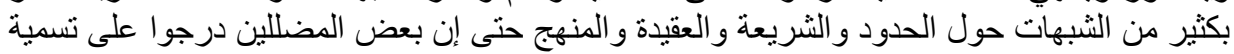

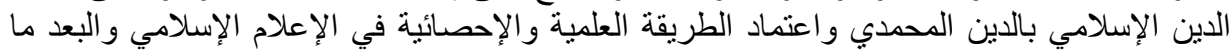

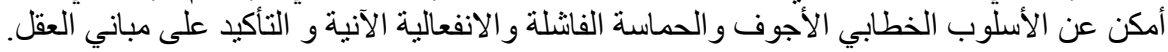

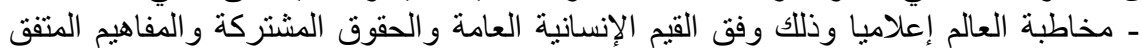

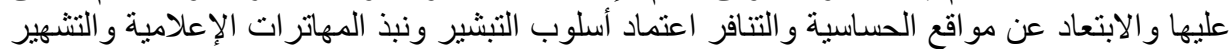

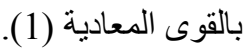

خاتمة

من خلال ما سبق يمكن أن نستتنج أن:

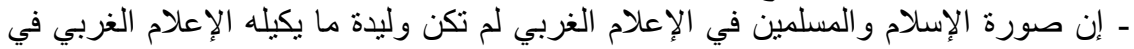

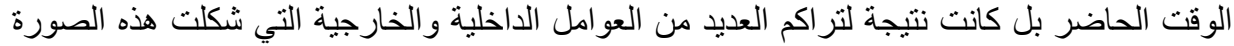
على مر العصور.

- وكما يتحمل الغرب جزء من المسؤولية في تثويه صورة الإسلام يتحمل المسلمون مسؤولية

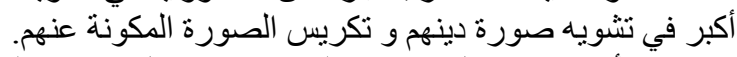

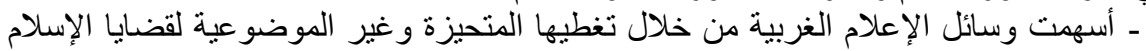

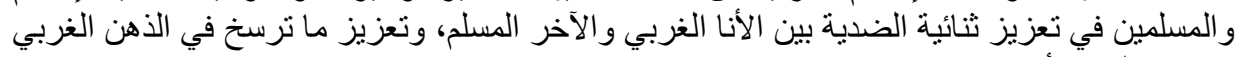

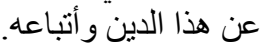
ـ لاز ال العامل الحضاري حاضر ا بقوة كو احد من اهم العو امل المحددة لصورة المسلم الآخر في

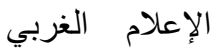

- يثكل موضوع النبي عليه الصلاة و السلام، الجهاد و الإرهاب و الخوف من الإسلام وقضايا

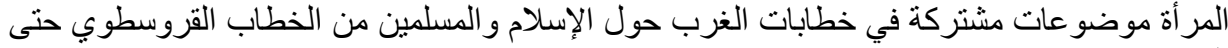

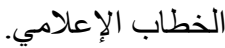




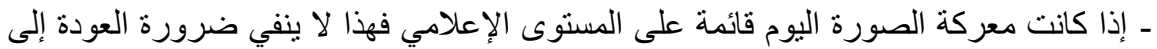

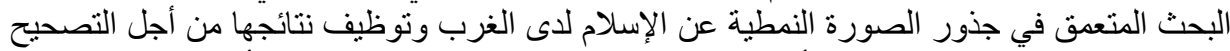
عبر وسائل الإعلام من جهة وكل الأدوات و الرو افد و الآليات المتاحة لذلك من جهة أخرى.

1. الإسلاحم و الغرب في مو اجهة الحملة الإعلامية الغربية ضد الإسلام و المسلمين، ط2، منشورات

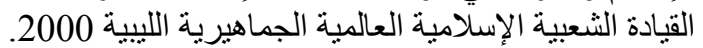

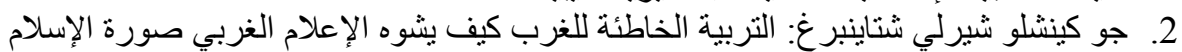

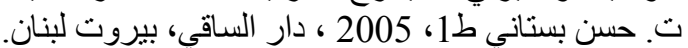

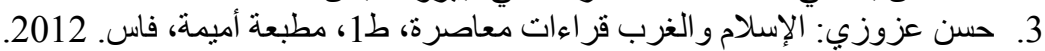

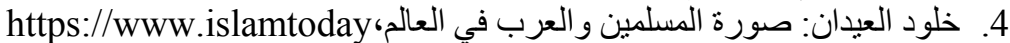

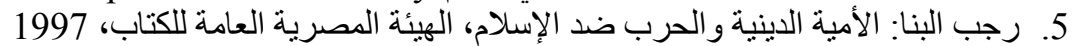
6. سعيد اللاوندي: الاسلاموفوبيا لماذا يخاف العرب من الاسلام، دار النهضة مصر للطباعة و لالانية النشر 2006 د ت ت.

7. سليمان سالم صالح: وسائل الإعلام و إدارة الصر اع العالمي، ط1، مكتبة الفلاح للنشر و التوزيع، 2011

8. سليمان صالح: وسائل الإعلام وصناعة الصور الذهنية، ط 1، مكتبة الفلاح للنشر والتوزيع،

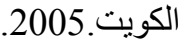

9. سماح صلاح: الجندر و الإعلام في الثرق الأوسط، ورقة عمل مقدمة لمركز وسائل الاتصال

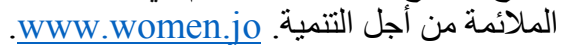
10. 10 علي خليل شقرة.

11.المبروك الثيباني المنصوري: صناعة الآخر المسلم في الفكر الغربي المعاصر من الاستشراف

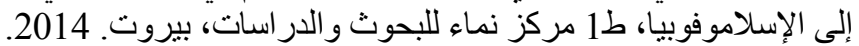

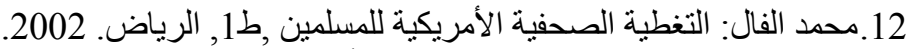
13.محمد عابد الجابري: الإسلام والغرب الأنابا والآخر، ط1، سلسلة فكر ونقد، الثبكة العربية

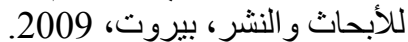

14.محي الدين عبد الحليم: الإعلام عن الإسلام في غير ديار الإسلام، د ط، الهيئة المصرية

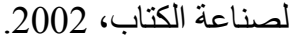

15.يحي العريضي: من بحكم أمريكا فعلا السيطرة الخفية لليهود دراسة في الإعلام الغربي

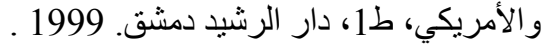

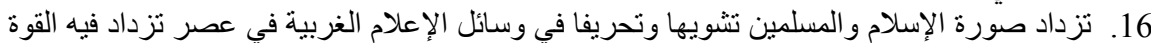

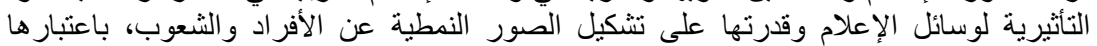

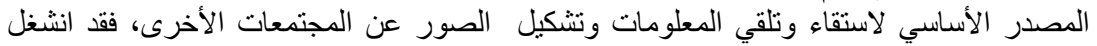

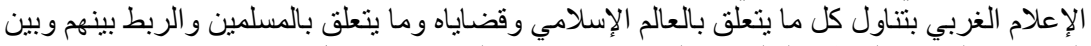

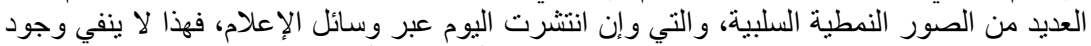

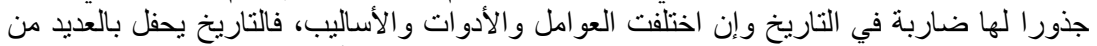

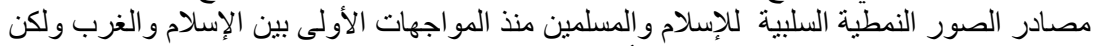

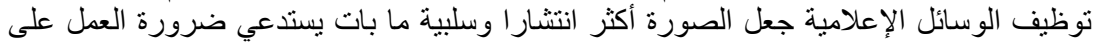

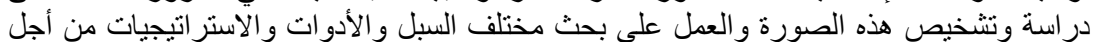




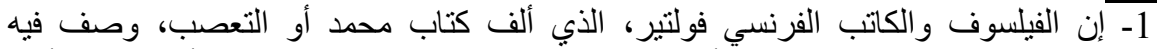

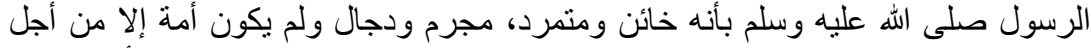

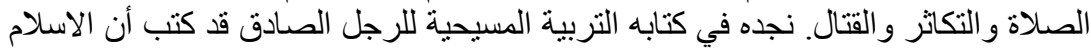

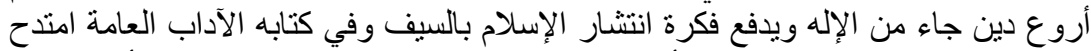

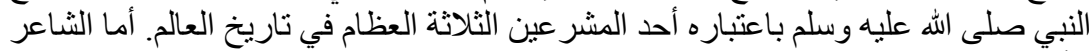

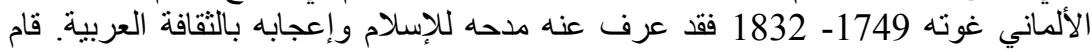

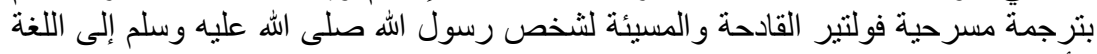

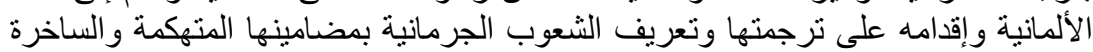

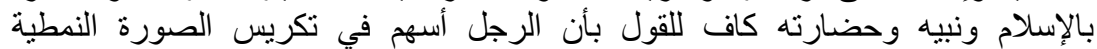

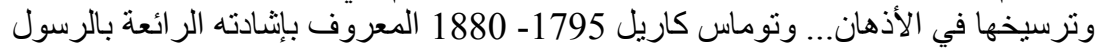

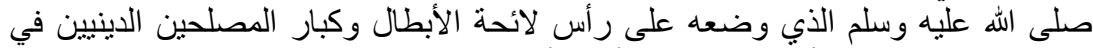

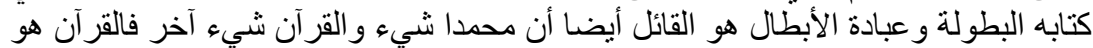

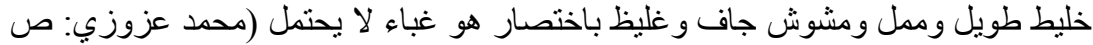
(281 279

2- الآخرية بصفتها مقولة فلسفية تعني في بعدها الفلسفي الوجودي أن من ليس أنا فهو آخر

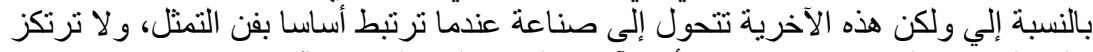

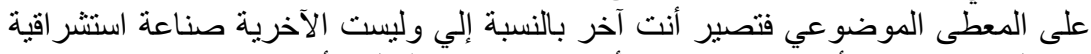

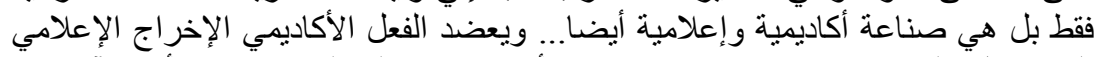

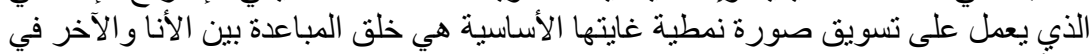
كل أُوجه الحياة. المبروك الثيقياني المنصوري: ص ص 13. 